\title{
The Effect of Electroacupuncture on Obesity through Browning of White Adipocytes and Mitochondrial Autophagy
}

MING WANG, MENGQIAN YUAN¹, ZHONGYUAN GUAN, ZHI YU² YANGGANG OU, WEI. GE AND XUANMING HU

Department of Acupunture and Massage, Geriatric Hospital Affiliated to Nanjing Medical University, No.30, Luojia Road, Nanjing, Jiangsu 210024, China; ${ }^{1}$ Department of Acupunture rehabilitation; ${ }^{2}$ Department of Acupunture and Medicine, ${ }^{3}$ Department of Acupunture, Jiangsu Provincial Hospital of Traditional Chinese Medicine, No.157, Daming Road, Nanjing, Jiangsu 210024, China

Wang et al:: Effect of Electroacupuncture on Obesity through Browning of White Adipocytes

To study the effect of electroacupuncture on obesity through browning of white adipocytes and mitochondrial autophagy. C57BL/6 mice were randomly divided into three groups, normal mice, obese mice and obese mice with electroacupuncture treatment (10 mice in each group). $2 / 15 \mathrm{~Hz}$ Electroacupuncture was applied to single Tianshu acupoint of obesity mice under conscious state for $15 \mathrm{~min}$ each time and 5 times/w for $4 \mathrm{w}$. Lee's Index and epididymal adipose cell size were measure to assess the therapeutic effect. Visceral white adipose tissue was Isolated and analyzed the expression level of uncoupling protein 1 and PR domain containing 16 by western blot. Messenger RNA of uncoupling protein 1, Peroxisome proliferator activated receptor gamma coactivator 1 alpha, PR domain containing 16; Beclin1, microtubule-associated protein light chain 3, phosphatase and tensin homolog induced putative kinase 1, Parkin and protein 62 were tested by quantitative real-time polymerase chain reaction. Average Lee's index of fat model group was significantly higher than that of control group $(p<0.05)$. Average Lee's index of electroacupuncture group was significantly lower compared to fat model group $(p<0.05)$. Epididymal adipose cell size of fat model group was significantly higher than that of control group $(p<0.05)$. Epididymal adipose cell size of electroacupuncture group was significantly lower than that of fat model group $(p<0.05)$. The relative gray value of uncoupling protein 1 and PR domain containing 16 of fat model group were both lower than that of the control group $(p<0.05)$. Compared to the fat model group, the electroacupuncture group had higher level of uncoupling protein 1 and PR domain containing $16(p<0.05)$. The mRNA of uncoupling protein 1 , Peroxisome proliferator activated receptor gamma coactivator 1 alpha, PR domain containing 16, Beclin1, microtubule-associated protein light chain 3, PTEN induced putative kinase 1, Parkin and protein 62 all decreased in the fat model group compared to the control group $(p<0.05)$. The electroacupuncture group raised the mRNA level of uncoupling protein 1, Peroxisome proliferator activated receptor gamma coactivator 1 alpha, PR domain containing 16, Beclin1, microtubule-associated protein light chain 3 , PTEN induced putative kinase 1, Parkin and protein 62 compared to the fat model group $(p<0.05)$. Electroacupuncture showed high efficacy on obesity for reducing Lee's index and epididymal adipose cell size of the obese mice. Electroacupuncture can trigger browning of white adipocytes to accelerate lipometabolism by increasing uncoupling protein 1 and PR domain containing 16. The other mechanism of electroacupuncture on obesity is mitochondrial autophagy by up-regulation of Peroxisome proliferator activated receptor gamma coactivator 1 alpha, beclin1, microtubule-associated protein light chain 3, phosphatase and tensin homolog induced putative kinase 1, Parkin and protein 62.

Key words: Electroacupuncture, obesity, browning of white adipocytes, mitochondrial autophagy

Pulmonary Obesity is a chronic disease with excessive accumulation or abnormal distribution of fat in body. As a high-risk factor of type 2 diabetes mellitus, cardiovascular disease and hypertension, obesity is showing a trend of rapid increase and younger age of morbidity. Currently, only calorie restriction is recommended for the treatment of obesity ${ }^{[1]}$. So far, have serious safety risks to patients such as cardiovascular

*Address for correspondence

E-mail: yichuifuou539@163.com 
and mental adverse events ${ }^{[2]}$, so there is a dilemma in anti-obesity drugs that is difficult to break through ${ }^{[3]}$. Besides anti-obesity drugs, there is another efficient choice for patient, which is called electroacupuncture. Electroacupuncture has lower side effects than antiobesity drugs, and can reduce the risk of a variety of complications ${ }^{[4]}$. However, further study for specific biological mechanism of electroacupuncture has not been elucidated clearly. This aim of this study was to study whether electroacupuncture can treat obesity by browning white adipocytes and inducing autophagy of adipocytes. Browning of white adipocytes is one of the important strategy in the treatment of obesity ${ }^{[5]}$, which has been proved to be a key role in the recovery of homeostasis of energy metabolism. There are two main types of adipocytes: white adipocytes and brown adipocytes. The main function of white adipocytes is storing energy, while brown adipocytes mainly consume energy. In recent years, it has been found that white adipocytes can consume energy through producing (uncoupling protein 1) UCP1, a marker of brown cells, and differentiate to brown cells under specific conditions. This process is called browning or beige.

New evidence indicated that browning of white adipocytes is regulated by mitochondrial autophagy ${ }^{[6]}$. Mitochondrial autophagy is a type of autophagy, which is an important regulatory process of energy metabolism balance. Mitochondrial remodeling and autophagy were observed in obese animal model by ultra-structural study, accompanied by the increase of mitochondrial autophagy inducer and Parkin, suggesting that energy stress of adipocytes can lead to mitochondrial autophagy and further cause abnormal energy metabolism of mitochondria ${ }^{[7]}$. After knockout of ATG7 (a yeast protein encoding gene necessary for mitochondrial autophagy) in mouse, the characteristics of white adipocytes became similar to brown adipocytes, with increasing mitochondrial number and enhanced lipid droplets autophagy (lipid droplets or adiposomes) mechanism ${ }^{[8]}$. In conclusion, mitochondrial autophagy plays an important role in regulating energy metabolism of adipocytes and inducing browning of white fat. We measure mRNA level of Bcl-2 homologous domain protein (Beclin1), microtubule-associated protein light chain 3 (LC3), phosphatase and tensin homolog (PTEN) induced putative kinase 1 (PINK1), Parkin and p62 in adipose for assess the effect of electroacupuncture to mitochondrial autophagy.Beclin1 and LC3 are important regulatory factors in autophagy. Beclin1, a homologous gene of yeast ATG6, participates in the formation of autophagosome by forming a complex with class III PI-3K, and plays an important role in the process of autophagosome membrane extension. LC3 protein can be divided into type I and type II. When autophagy occurs, type I LC3 is modified to form LC3 II. LC3 II protein is anchored on autophagy membrane, and its content is directly proportional to the number of autophagic vesicles, which can be used to evaluate the level of autophagy. PINK1/Parkin pathway is one of the most important pathways in mitochondrial autophagy pathway, and it is also considered as the most mature mechanism of mammalian mitochondrial autophagy ${ }^{[9]}$. Therefore, the expression of Beclin1, LC3, PINK1, parkin and p62 can be used as an important indicator for the regulation of autophagy.

C57BL/6 mice (male, $4 \mathrm{w}$ old, weighing about 10 g) were purchased from Yangzhou University and randomly divided into three groups, 10 mice in each group. Control group: normal diet without intervention Fat model group: After $30 \mathrm{~d}$ of feeding high-fat diet, the weight, length and obesity degree of mice were measured, and the model was successful constructed if the obesity degree was $20 \%$ more than the control group. Electroacupuncture group: base on the fat model, bipolar electroacupuncture was applied to single Tianshu acupoint of obesity mice under conscious state, and the frequency of electroacupuncture was $2 / 15 \mathrm{~Hz}$. Each acupoint conduct electroacupuncture for $15 \mathrm{~min}$ each time, and once a d, 5 times/w for $4 \mathrm{w}$. The mice were gently placed on the table top. Their posture was adjusted and stretched. The length between noses to anus was measured by the scale on the tape.

Lee's index $=3 \sqrt{ }$ bodyweight $\times 10^{3} /$ bodylength $(\mathrm{g} / \mathrm{cm})$. Mice in each group fasted overnight. The epididymal adipose tissue was rapidly separated on ice under anesthesia with $10 \%$ chloral hydrate. After washing with normal saline, the wet weight of adipose tissue was weighed. Frozen sections of tissue samples were stained with Immunohistochemical (IHC). Epididymal adipose tissue were fixed in $10 \%$ formalin at $4^{\circ}$ overnight and embedded in paraffin. $10 \mu \mathrm{m}$ sections of adipose tissue were cut and mounted onto gelatin-coated slides. The slides were added with HistoChoice for 15 min each for 2 times, followed by dehydration in $100 \%, 90 \%, 80$ $\%$ and $70 \%$ ethanol for $3 \mathrm{~min}$ respectively. The slides were stained with the hematoxylin for $60 \mathrm{~s}$. Then, the sections were rinsed in water for $15 \mathrm{~min}$, and stained with eosin for $30 \mathrm{~s}$. The sections were dehydrated in $100 \%$ ethanol for $5 \mathrm{~min}$ and incubation for $30 \mathrm{~min}$ in xylene. The diameter and perimeter of the two cells 
with the largest and smallest volume were selected under the same visual field by using dynamic images advanced 3.2 software. The mesenteric adipose tissue, bilateral perirenal adipose tissue and peri-epididymal adipose tissue were separated from mice body and washed with normal saline. Fresh tissue dissected from mice was washed with cold PBS and minced into small pieces. Most of the adipose tissues were stored at $-80^{\circ}$ after quick freezing, and a few of them were fixed with neutral formaldehyde solution. The VWAT samples of each group were lysed by Radio-Immunoprecipitation Assay (RIPA) buffer and determined concentration by bicinchoninic acid (BCA) method. The protein samples were incubated at $95^{\circ}$ for $5 \mathrm{~min}$ and were dissolved in ice bath for $30 \mathrm{~min}$. Then the protein was analyzed by SDS-PAGE electrophoresis and transferred into Polyvinylidene difluoride (PVDF) membrane. PVDF membrane was washed with PBS, and then incubated with blocking solution for $2 \mathrm{~h}$ at room temperature. Membrane was washed with PBS solution, and was sealed by $5 \%$ skimmed milk powder at room temperature. 1:1000 UCP1 polyclonal antibodies (abnova, CAT.NO.H00007350-A01-E) and 1:5000 PRDM16 antibody (Aviva Systems Biology, CAT.NO.ARP33260) was incubated with membrane overnight. Then the membrane was washed with phosphate buffer solution and horseradish peroxidase (HRP) labeled second antibody (diluted with 1:5000) was added. After incubation for $1 \mathrm{~h}$ at room temperature, the membrane was rinsed with TBST three times to remove the second antibody. The system was put into the chemiluminescence imaging system, and the gray value was read and analyzed by chemianalysis software.

Total ribonucleic acid (RNA) in VWAT was isolated with Trizol Reagent (GIBCO, CAT.NO. 15596-026) following the manufacturers protocol. Total RNAs were reverse transcripted into cDNA using miScript Reverse Transcription kit (Qiagen, CAT.NO.DXT-218061) at $26^{\circ}, 5 \mathrm{~min} ;\left(42^{\circ}, 30 \mathrm{~min} ; 86^{\circ}, 5 \mathrm{~min}\right) \times 40$ cycles. qRT-PCR analysis for UCP1, PGC1a, PRDM16,
Beclin1, Lc3, PINK1, Parkin and P62 was performed in Power SYBR GREEN PCR Master Mix (ABI, CAT. NO.4367659) in $20 \mu \mathrm{L}$ reactions respectively. The related mRNA expression levels were normalized to the $\beta$-actin by $2^{-\Delta \Delta C T}$. The primers were synthesized by Shanghai Sangon Company whose sequences were listed in Table 1. PCR program was $95^{\circ}: 30 \mathrm{~s}$, $\left(95^{\circ}: 5 \mathrm{~s}, 58^{\circ}: 35 \mathrm{~s}\right) \times 40$ cycles. SPSS v22.0 software was used for statistical analysis. Data were present as $(\mathrm{x} \pm \mathrm{s})$. Student's T-test was used for comparison between two groups, $p<0.05$ means there was statistical significance between different groups. Average Lee's index of fat model group was significantly higher than that of control group $(p<0.05)$, indicating that fat model was successful constructed. Average Lee's index of electroacupuncture group was significantly lower than that of fat model group $(\mathrm{p}<0.05)$, as shown in fig. 1 .

Average epididymal adipose cell size of fat model group was significantly higher than that of control group $(\mathrm{p}<0.05)$, also indicating that fat model was successful constructed. Epididymal adipose cell size of electroacupuncture group was significantly lower than that of fat model group $(p<0.05)$, as shown in fig. 2 . As

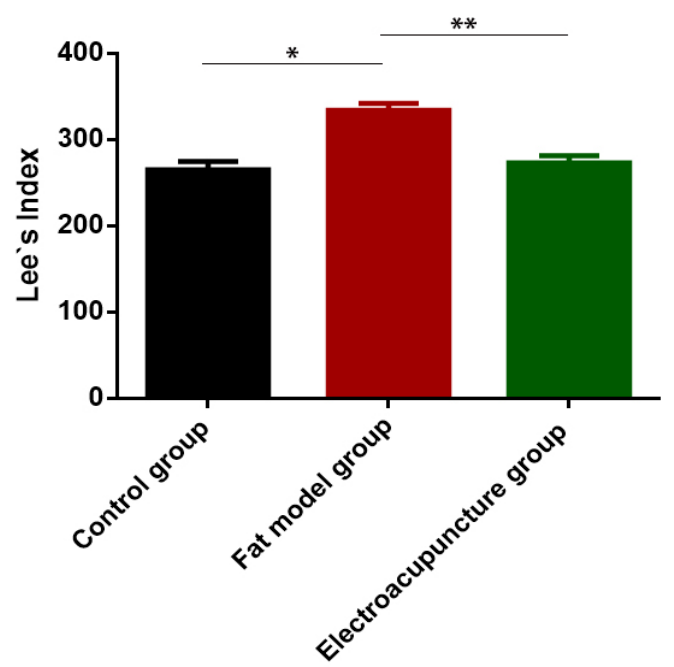

Fig. 1: Comparison of Lee's index of different groups ( $\mathrm{p}<\mathbf{0 . 0 5}$ vs control group, ${ }^{* *} \mathbf{p}<\mathbf{0 . 0 5}$ vs fat model group, $\mathrm{N}=10$ )

TABLE 1: THE PRIME SEQUENCES FOR QRT-PCR

\begin{tabular}{|l|r|r|}
\hline Gene & Forward primer sequence & Reverse primer sequence \\
\hline UCP1 & 5'-CCCTGGCAAAAACAGAAGGA-3' & 5'-AGCTGATTTGCCTCTGAATGC-3' \\
PGC1a & 5'-CCCTGGCAAAAACAGAAGGA-3' & 5'-AGCTGATTTGCCTCTGAATGC-3' \\
B-actin & 5'-AGCGAGCATCCCCCAAAGTT-3' & 5'-GGGCACGAAGGCTCATCATT-3' \\
PRDM16 & 5'-CGGAAGAGCGTGAGTACAAA-3' \\
Beclin1 & 5'-GGACCAGGAGGAAGCTCAGTACC-3' \\
LC3 & 5'-CAGGAGGAAGAAGGCTTGGTTAGC-3' \\
PINK1 & 5'-CAAGCAAGTGTCTGACCCAC -3' & 5'-GTCCGTGAACACCTTGACAC-3' \\
Parkin & 5'-CAGACAAGGACACATCAGTA -3' & 5'-TGTTGACCTTGGTCTGGTAGGAGAC-3' \\
p62 & 5'-TGGCCTGTGGATTTGTCGAC-3' & 5'-GACGACATCTGGGCCTTTTC-3' \\
\hline
\end{tabular}




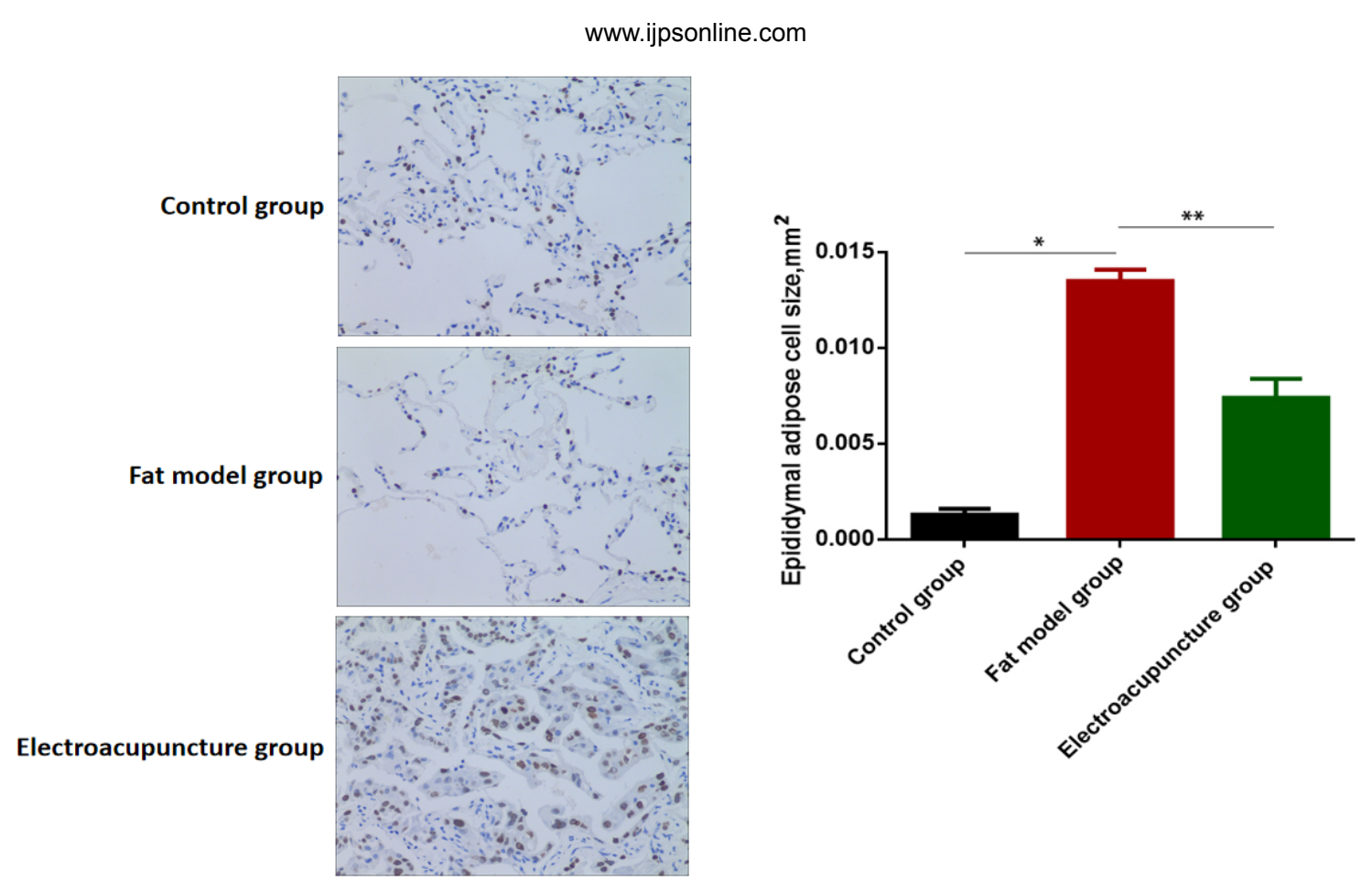

Fig. 2: The effect of electroacupuncture to epididymal adipose cell size $\left({ }^{*} \mathrm{p}<0.05\right.$ vs control group, ${ }^{* *} \mathrm{p}<0.05$ vs fat model group, $\left.\mathrm{N}=10\right)$
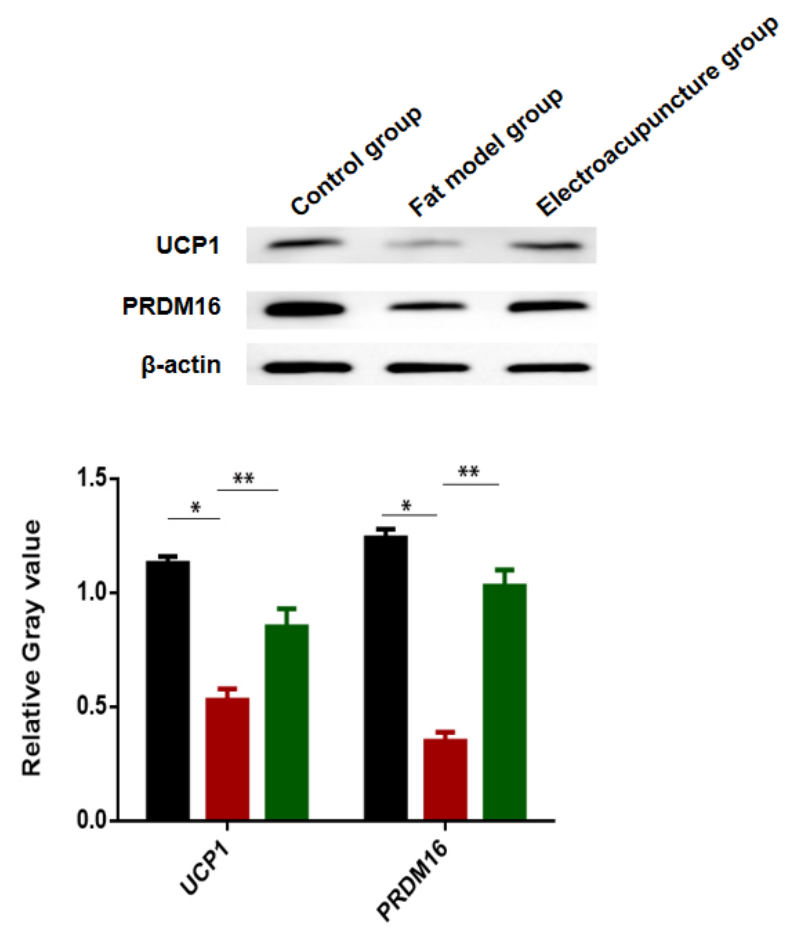

Fig. 3: The relative gray value of UCP1 and PRDM16 in different groups

( $\mathrm{p}<\mathbf{0 . 0 5}$ vs control group, ${ }^{* *} \mathrm{p}<0.05$ vs fat model group, $\mathrm{N}=10$ )

shown in fig. 3, the relative gray value of UCP1 and PRDM16 of fat model group were both lower than that of the control group $(\mathrm{p}<0.05)$. As biomarkers of brown adipocytes, decreasing UCP1 and PRDM16 in fat model suggested that obesity tend to differentiate more white adipocytes. Compared to the fat model group, the

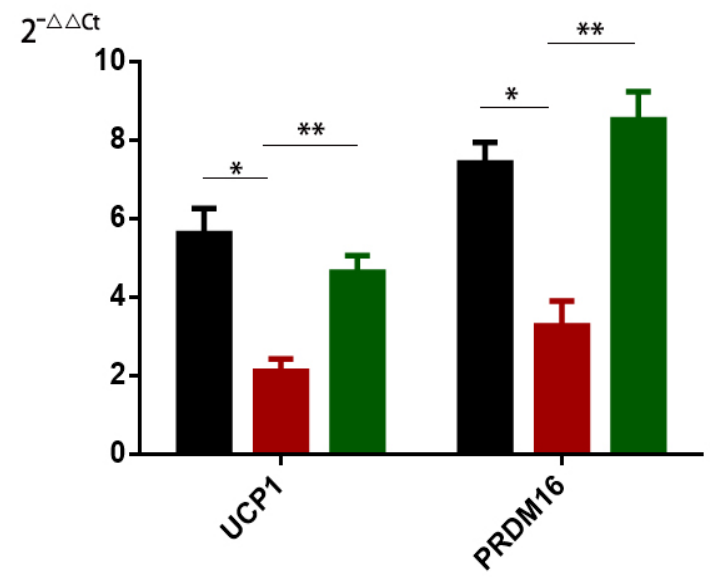

Fig. 4: The qPCR result of UCP1 and PRDM16 in different groups

( $\mathrm{p}<\mathbf{0 . 0 5}$ vs control group, ${ }^{* *} \mathrm{p}<\mathbf{0 . 0 5}$ vs fat model group, $\left.\mathrm{N}=10\right)$

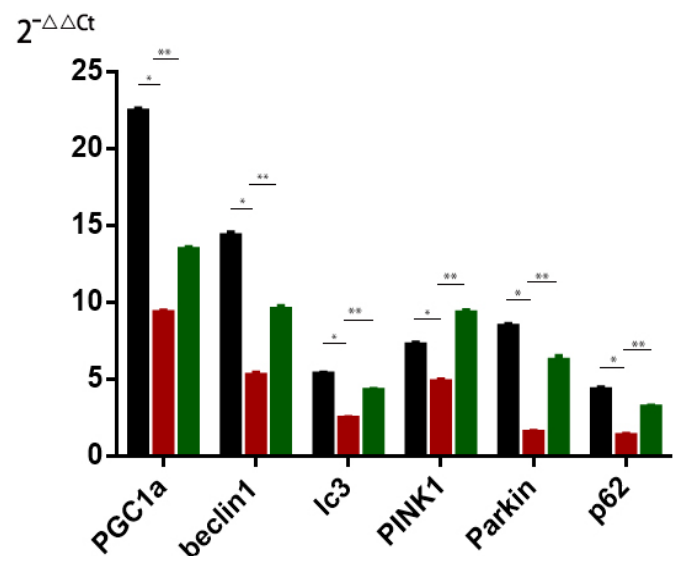

Fig. 5: The qPCR result of autophagy associated proteins in different groups

( $\mathrm{P}<\mathbf{0 . 0 5}$ vs control group, ${ }^{* *} \mathrm{P}<\mathbf{0 . 0 5}$ vs fat model group, $\mathrm{N}=10$ ) 
electroacupuncture group had higher level of UCP1 and PRDM16 $(\mathrm{p}<0.05)$.

qRT-PCR results showed that the mRNA of UCP1 and PRDM16 both decreased in the fat model group compared to the control group $(\mathrm{p}<0.05)$. The electroacupuncture group raised the mRNA level of UCP1 and PRDM16 compared to the fat model group $(p<0.05)$, as shown in fig. 4. The mRNA level of UCP1 and PRDM16 were corresponding to the protein content measured by qRT-PCR.

We also used q-PCR to analyze the mRNA level of autophagy associated proteins as PGC1a, beclin1, LC3, PINK1, Parkin and p62 in fat tissues. As shown in fig. 5, the mRNA level of PGC1a, beclin1, LC3, PINK1, Parkin and p62 all decreased significantly $(\mathrm{p}<0.05)$. After electroacupuncture treatment, the mRNA level of PGC1a, beclin1, LC3, PINK1, Parkin and p62 all increased in fat model group $(\mathrm{p}<0.05)$.

Obesity has become one of the main health problems worldwide. According to the epidemiological survey conducted in 195 countries around the world in 2015, a total of 603.7 million adults and 107.7 million children are obese, of which 70 countries have doubled their obesity population since $1980^{[10]}$. With the fast economic and social developments of China, The incidence of obesity in China shows an dramatic increasing trend in recent years, about $33.3 \%$ of Chinese adults aged more than 18 were with overweight or obesity ${ }^{[11]}$. Obesity and its complications result in heavy medical and society burden, obesity control and prevention strategies should be public health priorities in $\mathrm{China}^{[12]}$.

Electroacupuncture is a physiotherapy combined with tradition Chinese Acupoint Theory. Systematic review and meta-analysis showed that electroacupuncture can effectively reduce body weight, BMI and saddle width $^{[13]}$. One of the mechanisms of electroacupuncture on obesity is to inhibit insulin resistance in obese patients and regulate the expression of leptin and its receptor in the central nervous system, and then regulate the autocrine and metabolism of adipocytes ${ }^{[14]}$. Further experimental studies showed that electroacupuncture can regulate the expression of adenosine receptors A2AR in white adipocytes and, suggesting the effect of electroacupuncture on obesity is directly related to the activation of adipocyte metabolism ${ }^{[12]}$. We chose Tianshu acupoint for electroacupuncture because former study has proved that Tianshu acupoint can adjust intestinal and brain function to be norma ${ }^{[15]}$.

In this article, we found that electroacupuncture can reduce Lee's index of the obese mice to release the symptom and severity of obesity. Besides, epididymal adipose cell size became smaller after electroacupuncture. Electroacupuncture showed its favorable potential for treat obesity. For studying the mechanism of electroacupuncture on obesity, we used western blot and q-PCR to measure the content of UCP1 and PRDM16, two molecules that related to browning of white adipocytes. After electroacupuncture therapy, both mRNA and protein levels of UCP1 and PRDM16 were significantly higher than nontreatment mice, suggesting that electroacupuncture can promote lipid conversion and utilization through PRDM16 and UCP1. Electroacupuncture can trigger white adipocytes into brown adipocytes to accelerate lipometabolism. Mitochondria are important organelles for the consumption of excess nutrients in adipocytes. They are important sites for the metabolism of citric acid cycle, oxidative phosphorylation and fatty acid oxidation. Compared with white adipocytes, brown adipocytes have more mitochondria to oxidative phosphorylation, TCA cycle, and fatty acid $\beta$ oxidation and ketone metabolism. Moreover, the mRNA level of PGC1a, beclin1, LC3, PINK1, Parkin and p62 all increased significantly after electroacupuncture treatment. PGC1a, beclin1, LC3, PINK1, Parkin and p62 are biomarkers of mitochondrial autophagy that correlative to the activation of mitochondrial autophagy. Knockout of Beclin1 in adipocyte led to a hypertrophic enlargement of lipid droplets in brown adipocyte and impaired CL316243-induced lipolysis and energy metabolism ${ }^{[16]}$. LC3 conducted autophagymediated fat degradation to promote the assembly of the autophagic vesicles ${ }^{[17]}$. PINK1/Parkin pathway is the most recognized signaling pathway in mitophagy.

\section{Authors contributions:}

Wei Ge and Xuanming Hu conceived and designed the experiments; Mengqian Yuan, Zhongyuan Guan, Zhi $\mathrm{Yu}$ and Yanggang Ou performed the experiments; Ming Wang analyzed the data and wrote the paper.

\section{Acknowledgements:}

This work was supported by the National Natural Science Foundation of China (Grant No. 81904278). Wei $\mathrm{Ge}$ and Xuanming $\mathrm{Hu}$ are considered cocorresponding authors in our paper.

\section{Conflict of interests:}

The authors declared no conflict of interest. 


\section{REFERENCES}

1. Ryan DH, Kahan S. Guideline Recommendations for Obesity Management. Med Clin North Am 2018;102:49-63.

2. Krentz AJ, Fujioka K, Hompesch M. Evolution of pharmacological obesity treatments: focus on adverse sideeffect profiles. Diabetes Obes Metab 2016;18:558-70.

3. Squadrito F, Rottura M, Irrera N, Minutoli L, Bitto A, Barbieri MA, et al. Anti-obesity drug therapy in clinical practice: Evidence of a poor prescriptive attitude. Biomed Pharmacother 2020;128:110320.

4. Luo D, Liu L, Liang FX, Yu ZM. Electroacupuncture: A Feasible Sirt1 Promoter Which Modulates Metainflammation in Diet-Induced Obesity Rats. Evid Based Complementary Altern 2018;2018:1-10.

5. Thyagarajan B, Foster MT. Beiging of white adipose tissue as a therapeutic strategy for weight loss in humans. Horm Mol Biol Clin Investig 2017;31:16.

6. Altshuler-Keylin S, Shinoda K, Hasegawa Y, Ikeda K, Hong $\mathrm{H}$, Kang Q, et al. Beige Adipocyte Maintenance Is Regulated by Autophagy-Induced Mitochondrial Clearance. Cell Metab2016;24:402-19.

7. Cummins TD, Holden CR, Sansbury BE, Gibb AA, Shah J, Zafar N, et al. Metabolic remodeling of white adipose tissue in obesity. Am J Physiol Endocrinol Metab 2014;307:262-77.

8. Singh R, Xiang Y, Wang Y, Baikati K, Cuervo AM, Luu YK, et al. Autophagy regulates adipose mass and differentiation in mice. J Clin Invest 2009;119:3329-39.

9. Kubli DA, Zhang XX, Lee Y, Hanna RA, Quinsay MN, Nguyen CK, et al. Parkin Protein Deficiency Exacerbates Cardiac Injury and Reduces Survival following Myocardial Infarction. J Biol Chem 2013;288:915-26.

10. Song M. Trajectory analysis in obesity epidemiology: A promising life course approach. Curr Opinion Endocr Metab Res 2019;4:37-41.
11. Zhang LF, Wang ZW, Wang X, Chen Z, Shao L, Tian Y, et al. Prevalence of overweight and obesity in China: Results from a cross-sectional study of 441 thousand adults, 2012-2015. Obes Res Clin Pract 2020;14:119-26.

12. Zhang $\mathrm{K}$, Zhou S, Wang $\mathrm{C}, \mathrm{Xu} \mathrm{H}$, Zhang L. Acupuncture on Obesity: Clinical Evidence and Possible Neuroendocrine Mechanisms. Evid Based Complement Alternat Med 2018, 2018: 6409389.

13. Zhang RQ, Tan J, Li FY, Ma YH, Han LX, Yang XL. Acupuncture for the treatment of obesity in adults: a systematic review and meta-analysis. Postgrad Med J 2017;93:743-51.

14. Martinez B, Peplow PV. Treatment of insulin resistance by acupuncture: a review of human and animal studies. Acupunct Med 2016;34:310-19.

15. Jiang YJ. Clinical observation on Tui-pushing the Large Intestine Meridian plus point sticking at Tianshu (ST 25) in prevention of post-stroke constipation. J Acupunct Tuina Sci 2016;14:396-400.

16. Son Y, Cho YK, Saha A, Kwon HJ, Park JH, Kim M, et al. Adipocyte-specific Beclin1 deletion impairs lipolysis and mitochondrial integrity in adipose tissue. Mol Metab 2020;39:101005.

17. Zhang Y, Cui YZ, Wang L, Han JX. Autophagy promotes osteoclast podosome disassembly and cell motility athrough the interaction of kindlin3 with LC3. Cell Signal 2020;67:109505.

This is an open access article distributed under the terms of the Creative Commons Attribution-NonCommercial-ShareAlike 3.0 License, which allows others to remix, tweak, and build upon the work non-commercially, as long as the author is credited and the new creations are licensed under the identical terms

\begin{tabular}{l}
$\begin{array}{l}\text { This article was originally published in a special issue, } \\
\text { "Biomedical Research in Clinical and Preclinical } \\
\text { Pharmaceutics" Indian J Pharm Sci 2020:82(3)Spl issue7;67-72 }\end{array}$ \\
\hline
\end{tabular}

